\title{
CHANGES OF SOME BIOPHYSICAL VALUES IN ANIMALS UNDER MODERATE STRESS
}

\author{
Hristov Kr. \\ Dept. of Physiology and Pathophysiology, Division of Pathophysiology, Medical University - Varna
}

\author{
Reviewed by: assoc. prof. H. Bozov
}

\begin{abstract}
ASTRACT
In recent years, in the medical world the number of studies related to certain biophysical characteristics of biologically active points (similar to acupuncture points) - BAP, antenna and electromagnetic radiation effect of the body is increasing. As a product of nature, organisms come into simpler or more complex relationship with the various forms of manifestation of natural energy and particularly the electromagnetic one. Aim of this study was to identify changes in the antenna effect, electromagnetic radiation and resistance and semiconductor effect in BAP of experimental animals under oxidative stress response to hypobaric hypoxia and hyperbaric hyperoxia, cold and heat shock, physical exertion and prolonged immobilization. The general impression from the results obtained from different models of stress conditions, is that changes in biophysical parameters studied, with few exceptions in parameters and in varying degrees of variability are one-way and statistically significant. The increasing of antenna effect and electromagnetic radiation from one side and reducing resistance and of semiconductor effect in acupuncture point Yin-Tang considered for "basic energizer of the organism" at the other, in wide physical aspect are proof for an increase of communicative opportunities of animals under different stress opportunities. To build on the findings of experimental study it is necessary to extend the number of animals in the respective groups, and conduct further experiments with other stressful conditions.
\end{abstract}

Key words: stress, antenna effect, electromagnetic radiation, resistance, semiconductor effect, biologically active point, energetic communication

\section{BACKGROUND}

In recent years, in the medical world the number of studies related to certain biophysical characteristics of biologically active points (similar to acupuncture points) - BAP, antenna and electromagnetic radiation effect of the body is increasing $(3,5,6,7,8,9,10,11,18)$. Relatively little research are devoted to the changes that these characteristics suffer under the influence of various extreme external and internal factors $(1,2,12,15,19)$. In accordance with the empirical philosophical and religious notions of ancient Chinese medicine man (including other living organisms) "communicate" with the "energy" in nature and that communication depends on the condition of the body, stars, moon, sun etc. In terms of today's scientific knowledge, there is a chance for some explanation of these "communication" opportunities. Being product of nature, organisms come into simpler or more complex relationship with her, including various forms of manifestation of energy and particularly electromagnetic one. Regarding the electromagnetic energy it has

Address for correspondence:

Kr. Hristov, Dept. of Physiology and Pathophysiology, Division of Pathophysiology, Medical University - Varna 55 Marin Drinov str, 9002 Varna

e-mail: etlina@gmail.com been already shown that organisms have the antenna effect in the frequency range from 10 to $1000 \mathrm{MHz}(4,17)$. From technical point of view each antenna under certain conditions may not only to accept, but also to emit electromagnetic waves in the corresponding or close to that range. From the other side, in the literature in recent years biologically active points are characterized by reactance, capacitance, inductance, semiconductor effect and others $(14,16,20)$. Aim of this study was to identify changes in the antenna effect, electromagnetic radiation and resistance and semiconductor effect in BAP of experimental animals under oxidative stress response to hypobaric hypoxia and hyperbaric hyperoxia, cold and heat shock, physical exertion and prolonged immobilization.

\section{MATERIALS AND METHODS}

The study was conducted in 60 white inbreeded rats divided into 6 groups of 10 animals each. First group of animals was exposed to hyperbaric hyperoxia in hyperbaric chamber at absolute pressure of oxygen - $2700 \mathrm{hPa}$ for 30 minutes. A second group of animals was subjected to response to hypobaric hypoxia in hypobaric chamber for 30 minutes by reducing the total pressure in the chamber to $500 \mathrm{hPa}$, equivalent to $6000 \mathrm{~m}$ 
above sea level. A third group was placed in a freezer at a temperature of $-10^{\circ} \mathrm{C}$ for 30 minutes. Fourth group - placed in a heat chamber at $+50^{\circ} \mathrm{C}$ for 30 minutes. Fifth group - immobilization stress by fixing animals on their back and immobilizing the limbs and heads for 30 minutes. Sixth group was under moderate exercise, based on the method of Shiranyan and Avakyan for 15 minutes. For each animal were measured before and after the appropriate load the biophysical parameters of biologically active points (resistance and semiconductor effect), the antenna effect and electromagnetic radiation in the range of $100 \mathrm{MHz}$. For measuring the instruments used were as follows: digital multimeter Axiomet - AX-588, Trifield meter 100XE, antenna preamp Aerial booster - SB1-MP, biosensor detector of electromagnetic waves developed by us according to a classical electronic circuit $(4,13)$.

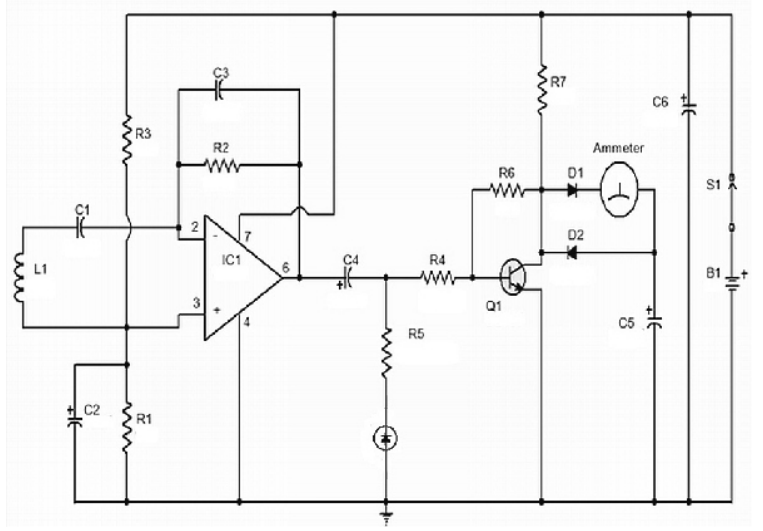

Pic. 1 Biosensor detector of electromagnetic waves

As a biologically active point the analogue of corporal point Yin Tang in rats was examined. This point is regarded in traditional Chinese medicine for major energizer of the body - the gateway to the temple in translation and is used primarily to treat the recovery of energy relationships in the body. The obtained results were processed by variance analysis.

\section{RESULTS AND DISCUSSION}

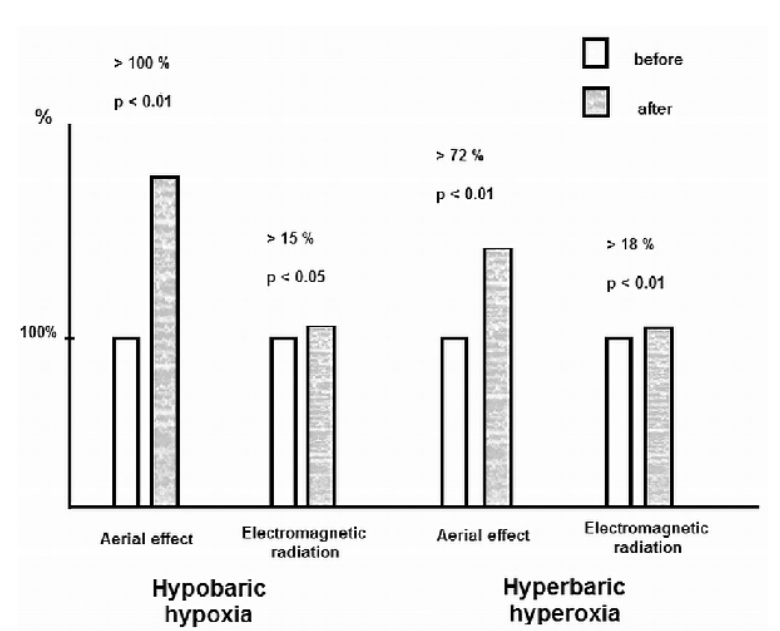

Fig. 1. Changes in antenna and electromagnetic radiation effect under oxidative stress.

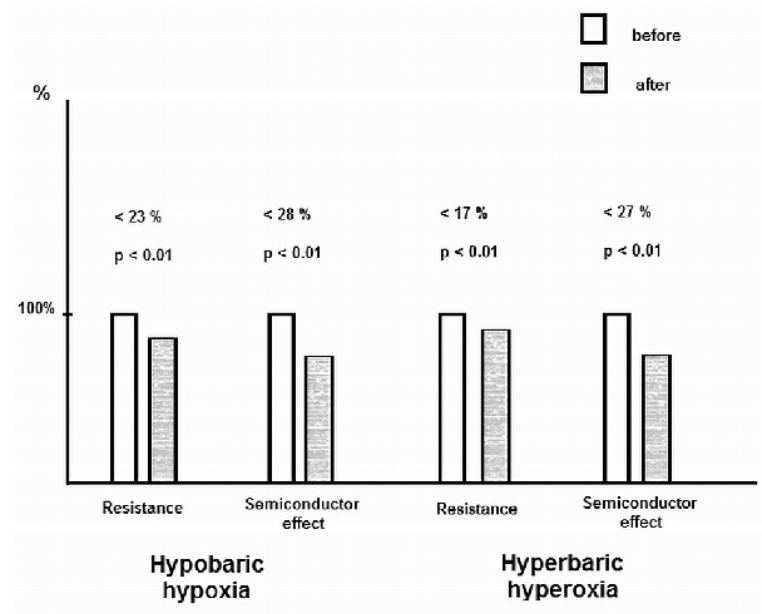

Fig. 2. Changes in resistance and semiconductor effect of acupuncture point Yin Tang under oxidative stress.

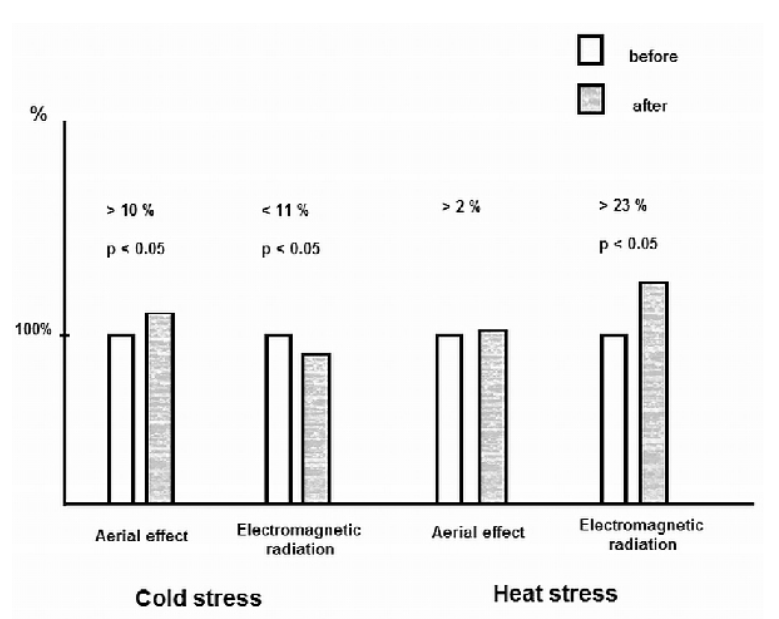

Fig. 3. Changes in antenna and electromagnetic radiation effect under thermal stress.

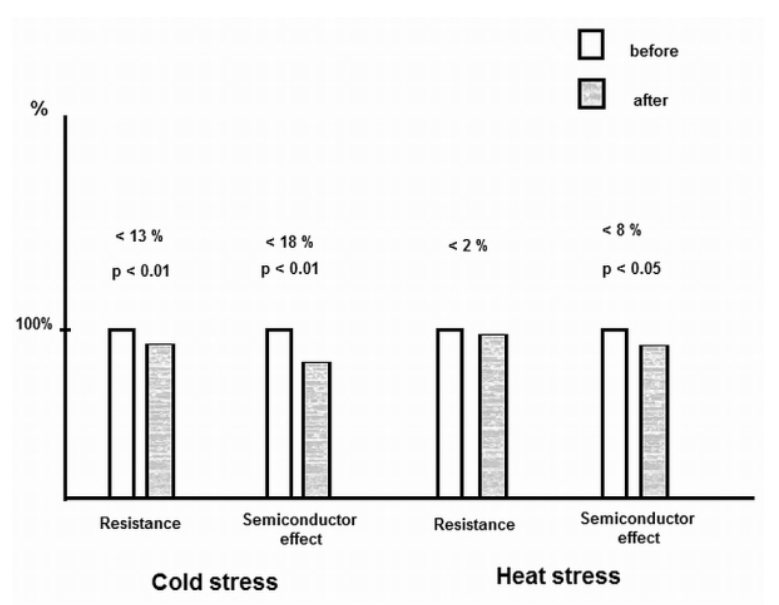

Fig. 4. Changes in resistance and semiconductor effect of acupuncture point Yin Tang under thermal stress

The results are exported in Figures 1 to 6:

The general impression from the results obtained in different models of stress conditions, is that changes in biophysi- 


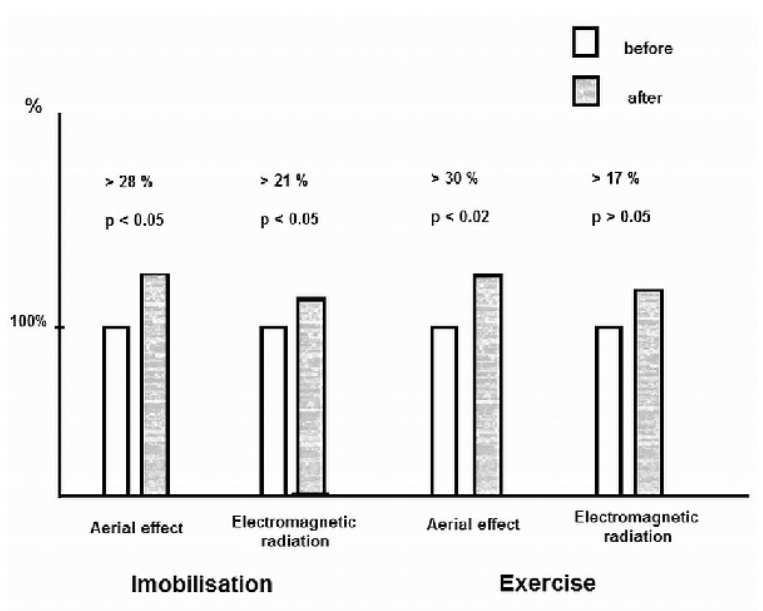

Fig. 5. Changes in antenna and electromagnetic radiation effect under immobilization stress and moderate exercise.

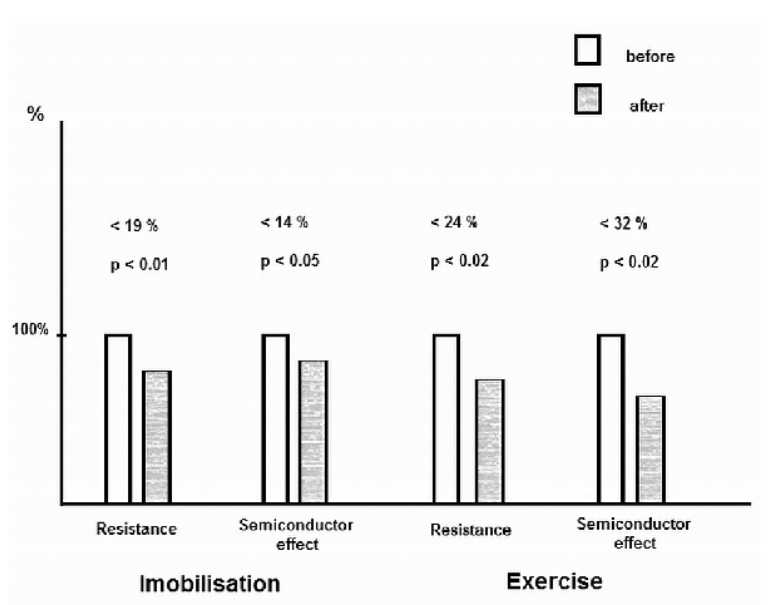

Fig. 6. Changes in resistance and semiconductor effect of acupuncture point Yin Tang under immobilization stress and moderate exercise.

cal parameters studied, with few exceptions in parameters and in varying degrees of variability, are one-way and statistically significant. The increase of antenna and electromagnetic radiation effect from one side and reducing resistance and the semiconductor effect in acupuncture point Yin-Tang considered for "basic energizer of the organism" on the other, in purely physical aspect are proof for increase of communicative opportunities of the animals placed in a different stress opportunity. Well-known fact in the physics of electromagnetic phenomena is that the reduction of the resistance leads to an increased conductivity and reducing of the semiconductor effect in practice leads to equalization of electromotive potentials in both directions of a structure. Despite the general applications of research under type of "Auto control", the results in particular animals are showing individual deviations from the average results for each group. Thus it is imperative that research be conducted with much larger groups of animals to achieve results with greater confidence.

\section{CONCLUSIONS}

Survey results to changes in biophysical parameters antenna effect, electromagnetic radiation and resistance and semiconductor effect of acupuncture point Yin Tang gives serious grounds, although not high statistical accuracy, to make even a preliminary conclusion as follows: Under conditions of oxidative stress, thermal stress, immobilization stress and physical activity to varying degrees for the particular model's conditions, the rats are responding by increased communication capabilities in the electromagnetic range of $100 \mathrm{MHz}$. To build on this finding it is necessary to expand the number of animals in the respective groups, and to conduct further experiments with other stressful conditions.

\section{BIBLIOGRAPHY}

1. Almeida RT, Duarte ID. Nitric oxide/cGMP pathway mediates orofacial antinociception induced by electroacupuncture at the St36 acupoint. Brain Res 2008; 1188: 54-60

2. Andersson S, Lundeberg T. Acupuncture from empiricism to science: functional background to acupuncture effects in pain and disease. Med Hypotheses 1995;45:271-281.

3. Balanis C.A., "Horn Antennas," in Antenna Handbook; theory, applications, and design, Y.T. Lo and S.W. Lee, edilors, Van Nostrand Reinhold, 1988, page 8-39.

4. Beverly Rubik, Measurement of the Human Biofield and Other Energetic Instruments, Chapter 20 of Energetics and Spirituality by Lyn Freeman, 2008

5. Croley E., Electrical acupuncture point conductance in the living compared to that in the dead. Amer. J. of Acupuncture, 14 (1): 57-60, 1986.

6. Ho, M.W. and P.T. Saunders. Liquid crystalline mesophases in living organisms. in Bioelectrodynamics and Biocommunication, Ho,M.W., F.A. Popp and U. Warnke (Eds.), World Scientific, Singapore, 1994.

7. Kirilov J. Needle antenna properties in acupuncture. Needle inducing mechanism on biological active points - IV Symp. "New in theory and practice of NBO" 1989,Moscow.

8. Laszlo, E. The Interconnected Universe, World Scientific, Singapore, 1995.

9. Mayer DJ. Biological mechanisms of acupuncture. Prog Brain Res 2000;122:457-477.

10. Myeong Soo Lee, Sang-Yong Jeong, Yong-Heum Lee et al., Differences in Electrical Conduction Properties Between Meridians and Non-meridians. - The American Journal of Chinese Medicine (An International Journal of Comparative Medicine East and West), 33, 5: 723-728, 2005.

11. Oschman, J.L.: A Biophysical Basis of Acupuncture, Private manuscript, 1993.

12. Portnov F. Electro-puncture reflex-therapy. - Riga, Zinatne, 1987, 352 p (rus).

13. Robert Mailoux, Antenna handbook, Artech house, London, 2005 
14. Sancier K M: Electrodermal measurements for monitoring the effects of a qigong workshop, $J$ Altern Complement Med 9(2):235, 2003.

15. Sator-Katzenschlager SM, Szeles JC, Scharbert G, Michalek-Sauberer A, Kober A, Heinze G, Kozek-Langenecker SA. Electrical stimulation of auricular acupuncture points is more effective than conventional manual auricular acupuncture in chronic cervical pain: a pilot study. Anesth. Analg. 2003; 97:1469-1473.

16. Teppone M., Avakyan R., Can a modern doctor understand traditional Chinese medicine? In: Theoretical and clinical aspects of Bio-resonance and multi-resonance therapy application: The X International Congress, Moscow, 2004, part II, pp. 395-400 (rus).
17. Victor J. Stenger, Bioenergetic Fields, Scientific Review of Alternative Medicine, Vol. 3, No. 1, Spring/Summer 1999.

18. Wang, K. and J. Liu. Needling sensation receptor of an acupoint supplied by the median nerve - studies of their electro-physiological characteristics. American Journal of Chinese Medicine 17: 145-155, 1989.

19. White PF, Craig WF, Vakharia AS, Ghoname E, Ahmed HE, Hamza MA. Percutaneous neuromodulation therapy: does the location of electrical stimulation effect the acute analgesic response? Anesth Analg 2000;91:949-954.

20. Wu Jing, Van Yushen, A Hundred answers to the questions about "Zhou Yi" (transl. from chinese) Kiev, Nika-Center, 2001, 312 p (rus). 